\title{
Reliable Event Detection for Incomplete and Streaming (S)TEM Images
}

\author{
Sarah Reehl ${ }^{1}$, Bryan Stanfill ${ }^{1}$, Danny Ries ${ }^{2}$, Maggie Johnson ${ }^{2}$, Layla Mehdi ${ }^{1}$, Nigel D. Browning ${ }^{1}$, Lisa \\ Bramer $^{1}$ \\ 1. Pacific Northwest National Laboratory, Richland USA \\ 2. Iowa State University, Department of Statistics, Ames USA
}

A scanning transmission electron microscope (STEM) scans a specimen with a concentrated beam of electrons. Large doses of electrons provide high resolution images, but at the cost of damaging the specimen. A promising approach to mitigate specimen damage is to expose only randomly selected areas of the specimen to the electron beam $[1,2,3]$. Immediate qualitative analysis of subsampled images is impossible, however, as the subsampled images are difficult to interpret by the microscope operator (Figure 1). Therefore, without an additional tool to aid the operator in steering the microscope when the specimen is subsampled, the idea of random sampling is of theoretical promise, but of limited use in practice.

Though subsampling reduces the amount of data to process, the volume and velocity of the data produced by the electron microscope experiments pose a real problem. Subsampling the specimen results in images with much fewer pixels, e.g. 100,000, but these incomplete images are produced at a faster rate, which compounds the velocity issue. Therefore, any methodology used to analyze a STEM experiment in real or near real time must be able to accommodate incomplete data of high volume and velocity. To our knowledge, no existing image processing techniques can fulfill all three of these requirements.

We address these challenges and provide a methodology for online event detection on subsampled STEM images. Some examples of events we might detect are microscope drift, material growth, and change in size or appearance of nanoparticles. During live experiments we determine a baseline set of images and quantify the distance between newly acquired images and the established baseline. Using statistical methods, we capture localized significant changes in the images and allow enough flexibility to either establish a new baseline, depending on the phase of the experiment, or continue to monitor changes with respect to the beginning of the experiment.

In this presentation we will cover the development and deployment of the $\boldsymbol{R}$ eal-time $\boldsymbol{E}$ vent $\boldsymbol{D}$ etector for Subsampled Images (REDSI), a tool that provides microscopists with real-time data analysis support for their experiments. REDSI helps the microscopist steer a STEM experiment by providing them with streaming feedback about how and where the specimen being imaged is changing. REDSI is applicable when the specimen is fully or sparsely sampled, which brings the theoretical advantages of subsampling into the realm of possible solutions. We also demonstrate the use of REDSI applied to live TEM experiments (Figure 2) [4]. 


\section{References:}

[1] L. Kovarik, A. Stevens, A. Liyu et al. Appl Phys Lett 109(16), (2016), p. 164102.

[2] A. Stevens, H. Yang, L. Carin et al. Microscopy 63(1), (2014), pp. 41.

[3] A. Béché, B. Goris, B. Freitag et al. Applied Physics Letters 108(9), (2016), p. 093103.

[4] Supported by the Chemical Imaging, Signature Discovery, and Analysis in Motion Initiatives at PNNL. PNNL is operated by Battelle Memorial Inst. for the US DOE; contract DE-AC05-76RL01830.
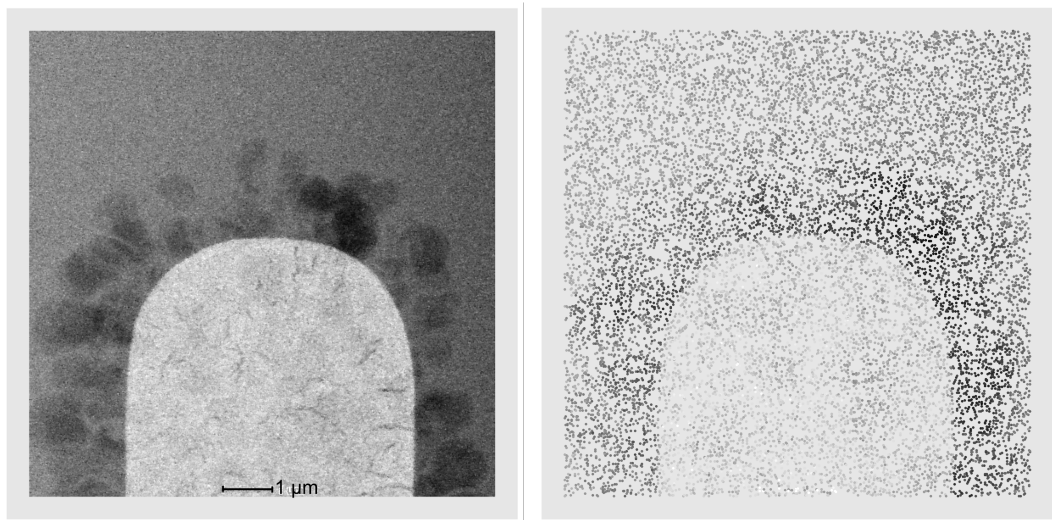

Figure 1. A complete (left) and sub-sampled (right) high-angle angular dark field (HAADF) image depicting anode (lightest pixels), lithium dendrite growth (darkest pixels), and electrolyte background. The sub-sampled image contains $5 \%$ of the complete image.

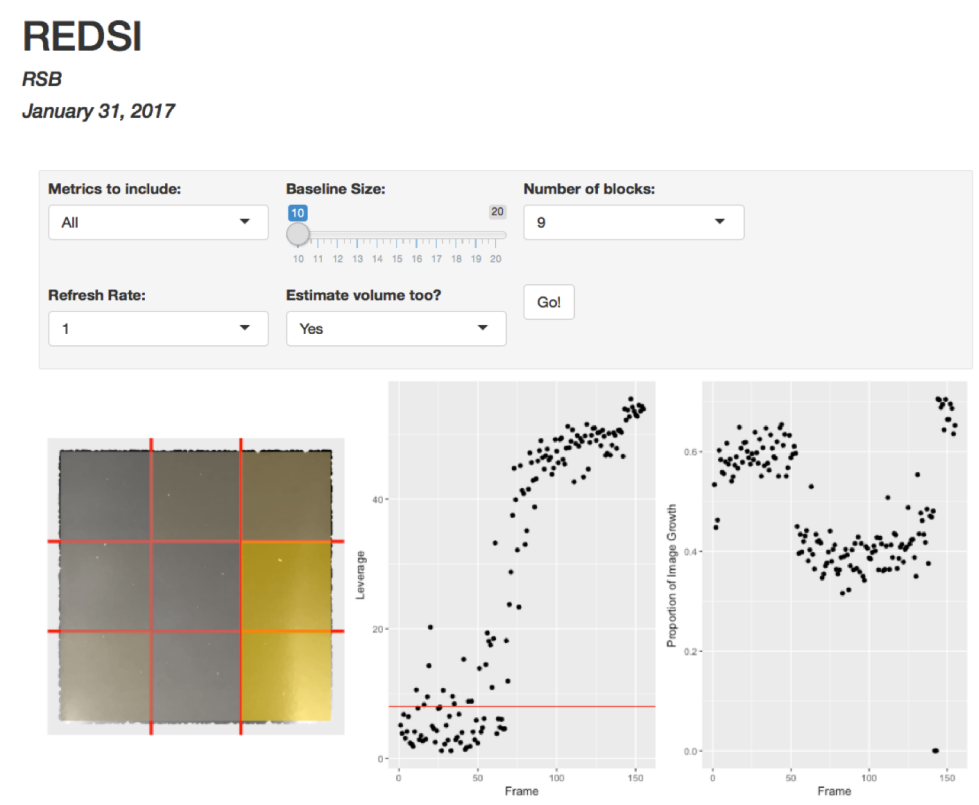

Figure 2. REDSI deployed on an electron microscope during a STEM experiment at PNNL. This method uses localized event detection to highlight changing regions of newly acquired images (left), a global score to indicate the magnitude of the change (center), and a quantitative material growth estimate (right). 